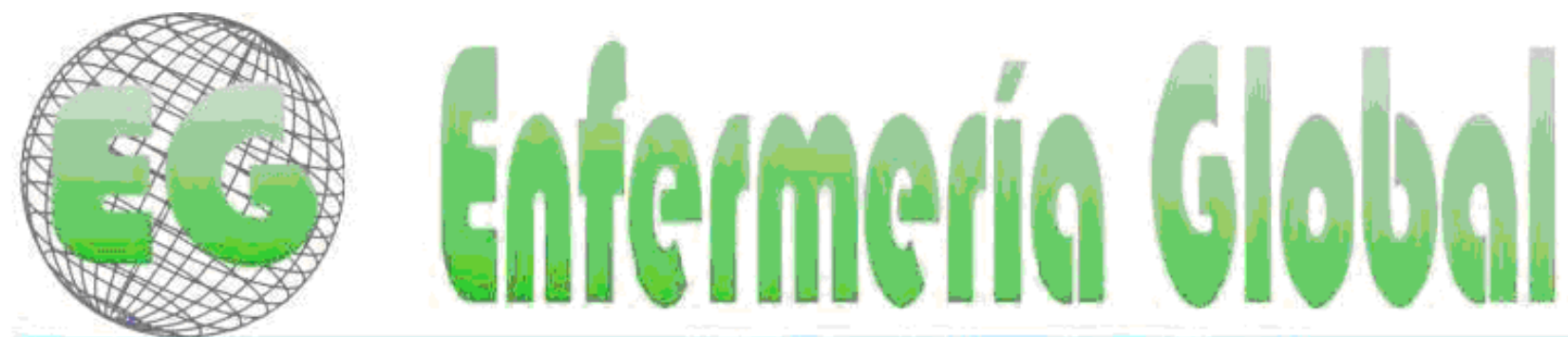

$19 \operatorname{SN} 1695-6144$

$\mathrm{N}^{\circ} 16$

Revista electrónica cuatrimestral de Enfermería

Junio 2009

www.um.es/eglobal/

DOGENGIA - FORMACIÓN

\title{
EFICACIA DE UN PROGRAMA EDUCATIVO AL PACIENTE INGRESADO CON NEUMONÍA
}

\section{EFFICIENCY OF AN EDUCATIONAL PROGRAM FOR THE INPATIENT WITH PNEUMONIA}

*Estrada Reventos, D., *Lara Pulido, A., "*Robau Gassiot, M., " Casals Font, E., ${ }^{* * *}$ Fernández Martínez, A., ${ }^{* * *}$ Rosell Marti, M.

*Unidad de Medicina Interna.**Unidad de Infecciones.***Unidad de Neumología. Hospital Clínico de Barcelona

Palabras clave: neumonía, información, educación, seguridad paciente

Keywords: Pneumonia, Information, Education, Patient Safety.

\section{RESUMEN}

Objetivo: Elaboración de un folleto informativo sobre la neumonía, en forma de tríptico, dirigido a pacientes ingresados por este motivo, así como a sus familiares y cuidadores y su posterior evaluación.

Material y método: El estudio consta de dos partes. 1a Estudio observacional: revisión bibliografíca de la neumonía en bases de datos internacionales, elaboración de un tríptico estructurado y comprensible para el paciente que incluye definición, síntomas, cómo se diagnostica y tratamiento. $2^{\mathrm{a}}$ Estudio experimental, cualitativo y prospectivo en dos grupos de pacientes ingresados con neumonía adquirida en la comunidad en un hospital de tercer nivel. En cada grupo entraron 38 pacientes. El grupo intervención recibió el folleto informativo en forma de tríptico y el grupo control no recibió ninguna intervención. A ambos grupos se les pasó un cuestionario al alta con preguntas relacionadas sobre la neumonía.

Resultado: Muestra de 76 pacientes, 58\% hombres con una edad media de 69 años y un nivel de estudios primarios de un $50 \%$. El $79 \%$ era la primera vez que tenia neumonía. En el estudio comparativo de los dos grupos destaca que en el grupo intervención se le había explicado lo que era la neumonía al $100 \%$ de pacientes mientras que en el de control era del $52 \%$. Referente a la pregunta de cuáles son los síntomas que aparecen en la neumonía en el grupo intervención el 99\% es capaz de enumerarlos, sin embargo, en el grupo control solo un $56 \%$ es capaz.

Conclusión: Consideramos que la importancia de la información facilitada por escrito, con un lenguaje claro, conciso y asequible para las diferentes edades en pacientes ingresados, aumenta sus conocimientos sobre su enfermedad, síntomas y pruebas, obteniendo más confort y tranquilidad durante su estancia. 


\section{ABSTRACT}

Objective: Elaboration of an informative three-page leaflet on pneumonia, directed toward patients hospitalized for this illness, as well as to their relatives, care takers and posterior evaluation.

Material and Method: The study consists of two parts. First, an observational study: Bibliographic review of pneumonia based on international information, elaboration of a constructed and understandable triptych for the patient which includes definition, symptoms, and information on diagnosis and treatment. Second, experimental, qualitative and prospective study in two groups of patients hospitalized with pneumonia acquired in their community in a third level hospital. There were 38 patients in each group. The group having an operation received the information leaflet in the form of a triptych and the control group did not have any operation. Both groups answered a questionnaire at discharge with questions related to pneumonia.

Results: Sample of 76 patients, $58 \%$ being men with an average age of 69 years and $50 \%$ with a level of primary education. A percentage of $79 \%$ had pneumonia for the first time. In the comparative study of the two groups it was stressed that the intervention group had completely been advised as to what pneumonia is while $52 \%$ in the control was advised. Regarding the question of what the symptoms that appear in pneumonia in the intervention group were, $99 \%$ were able to list them, but in the control group only $56 \%$ were capable.

Conclusion: We believe that the importance of the information provided in writing, with clear, concise and accessible language for hospitalized patients of different ages, increases their knowledge of their disease, symptoms and testing, obtaining more comfort and peace of mind during their stay.

\section{INTRODUCCIÓN}

La neumonía adquirida en la comunidad, es una de las enfermedades infecciosas más frecuentes y un motivo importante de morbilidad y mortalidad hospitalaria, con un gran impacto económico en el campo hospitalario y extrahospitalario ${ }^{1-2}$.

Representa entre el 30 y $60 \%$ de infecciones que precisan hospitalización ${ }^{3}$. En España según un estudio con base poblacional la incidencia fue de 1,6 casos/1000 adultos/año, de los cuales un $61.4 \%$ requirió ingreso ${ }^{4}$. Además un $8 \%$ de los pacientes que ingresan deben hacerlo directamente en las unidades de cuidados intensivos, con una tasa de fallecimientos que oscila entre el 21 y 58\%, mortalidad que ha aumentado en los últimos años ${ }^{5}$.

La información que reciben los pacientes, sus familiares y cuidadores acerca de su estado de salud es un aspecto primordial, ya que determinará en gran medida el grado de aceptación de su situación y la adherencia al tratamiento. Cuanto más informados estén, mucho más fácil, cómodo y agradable será su proceso durante su hospitalización. Esta información debe darse con un mensaje claro y sencillo. Según algunos estudios un paciente informado es un paciente mucho más seguro ${ }^{6}$.

\section{OBJETIVOS}

Elaboración de un programa educativo en forma de tríptico dirigido a pacientes ingresados con neumonía, así como a sus familiares y cuidadores con la función de aumentar su información e implicación en su proceso.

Analizar la eficacia de este programa educativo de intervención. 


\section{MATERIAL Y MÉTODOS}

Han participado tres unidades del Hospital Clínico de Barcelona, (medicina interna, neumología y enfermedades infecciosas) con similares recursos humanos y técnicos.

El trabajo consta de dos partes:

1를 Revisión bibliográfica de la neumonía en base de datos internacionales ${ }^{7-8}$, elaboración de un tríptico estructurado y comprensible para el paciente con dibujos y frases cortas, que incluye definición, síntomas, cómo se diagnostica y tratamiento ${ }^{9}$.

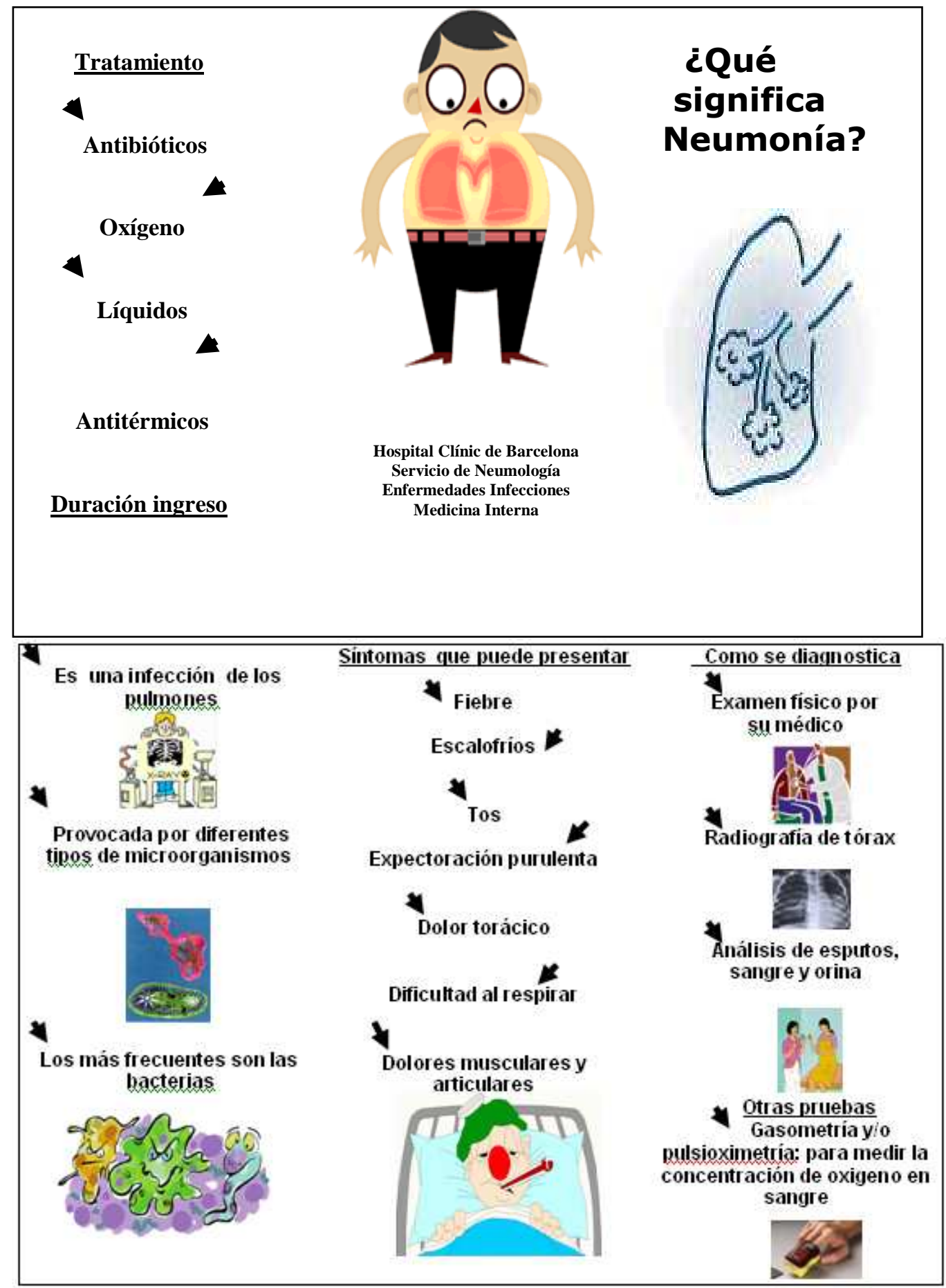

$2^{\text {a }}$ Estudio experimental, comparativo y prospectivo en dos grupos de pacientes ingresados con neumonía adquirida en la comunidad en el Hospital Clínico de Barcelona. El grupo 
intervención recibió a las $24 \mathrm{~h}$ de ingreso un programa educativo escrito (tríptico) y oral mediante la explicación del tríptico por parte de de la enfermera encargada del paciente y el grupo control no recibió ninguna intervención. Al alta a ambos grupos se les pasa un cuestionario (Ver anexo 1) con 7 preguntas de formato cerrado relacionadas con la neumonía ${ }^{10}$. A ambos grupos se les propuso colaborar de forma voluntaria en el estudio. Se realizó un estudio piloto con 5 pacientes antes de iniciar el estudio global para comprobar la calidad técnica del cuestionario y valorar la comprensión de las preguntas.

Fueron excluidos del estudio los pacientes con deficiencias psíquicas y patologías médicas graves y sin ningún familiar o cuidador que pudieran contestar a la encuesta y a los pacientes con neumonía hospitalaria.

Se incluyeron de forma consecutiva y aleatorizada todos los pacientes que ingresaron en estas tres unidades con el diagnóstico de neumonía adquirida en la comunidad, entre febrero y mayo del 2008. La asignación a uno u otro grupo se realizó de forma aleatoria por un sistema informático de numeración y letra: A (intervención) o B (no intervención).

Los datos de las variables estudiadas se introdujeron en una base de datos y se analizaron mediante el programa estadístico SPSS para Windows (versión 12.0).

\section{RESULTADOS}

En el período comprendido entre febrero y mayo del 2008 la participación de pacientes fue similar en las tres unidades (neumología, medicina interna y enfermedades infecciosas) con un total de 76 pacientes, 38 fueron al grupo control y 38 al grupo intervención.

Por sexos, eran hombres un $60 \%$ en el grupo control y un 55\% en el grupo intervención. Referente a la edad no mostraron diferencias entre sí, con una edad media de 68 años en los pacientes del grupo control y 69 en los pacientes del grupo intervención, con limites de 25 y 98 años.

El nivel educacional mayoritario fue el del los estudios primarios en un $50 \%$, seguido del de estudios de bachillerato en un $20 \%$. (Tabla 1).

Tabla 1 Nivel de estudios de la persona que responde

\begin{tabular}{|l|l|l|l|l|l|l|}
\multicolumn{4}{c}{ total } & \multicolumn{3}{c|}{ intervención } \\
\hline Ninguno & 7 & $9 \%$ & 3 & $8 \%$ & 4 & $10 \%$ \\
\hline Primarios & 38 & $50 \%$ & 19 & $50 \%$ & 19 & $50 \%$ \\
\hline $\begin{array}{l}\text { Formación } \\
\text { profesional }\end{array}$ & 6 & $8 \%$ & 2 & $6 \%$ & 4 & $10 \%$ \\
\hline Bachillerato & 20 & $20 \%$ & 7 & $18 \%$ & 8 & $22 \%$ \\
\hline Universitarios & 13 & $13 \%$ & 7 & $18 \%$ & 3 & $8 \%$ \\
\hline
\end{tabular}

Los cuestionarios mayoritariamente un $96 \%$ fueron respondidos por el mismo paciente. El $79 \%$ de los pacientes que contestaron a la encuesta era la primera vez que ingresaban por neumonía.

Respecto a la pregunta ¿le han explicado qué es la neumonía?, en el grupo intervención se le había explicado en un $100 \%$ mientras que en el grupo control era del $52 \%$. 
Referente a la pregunta que respondió el grupo intervención sobre ¿Entendió la información que le dieron en forma de tríptico? El $69 \%$ lo entendió mucho y el $31 \%$ bastante.

En cuanto a la pregunta "La información que le han dado sobre su enfermedad (neumonía) y pruebas le parece adecuada" en el grupo intervención la respuesta fue que un $60 \%$ lo entendió mucho y un $40 \%$ bastante, mientras que en el grupo control un $50 \%$ poco y solo un $5 \%$ mucho.

Relativo a la pregunta ¿Qué cree usted que es la neumonía? en el grupo intervención un $100 \%$ respondió que era una infección, mientras que el grupo control respondió un $73 \%$ infección, $16 \%$ agua y $11 \%$ obstrucción.

En la última pregunta ¿Cuál de estos síntomas aparece en la neumonía? hubo tres respuestas adecuadas, en el grupo intervención un 99\% es capaz de enumerar las tres, mientras que en el grupo control solo han sido capaces un $56 \%$. (Tabla 2 ).

\section{Tabla 2 ¿Cuál de estos síntomas aparece en la neumonía?}

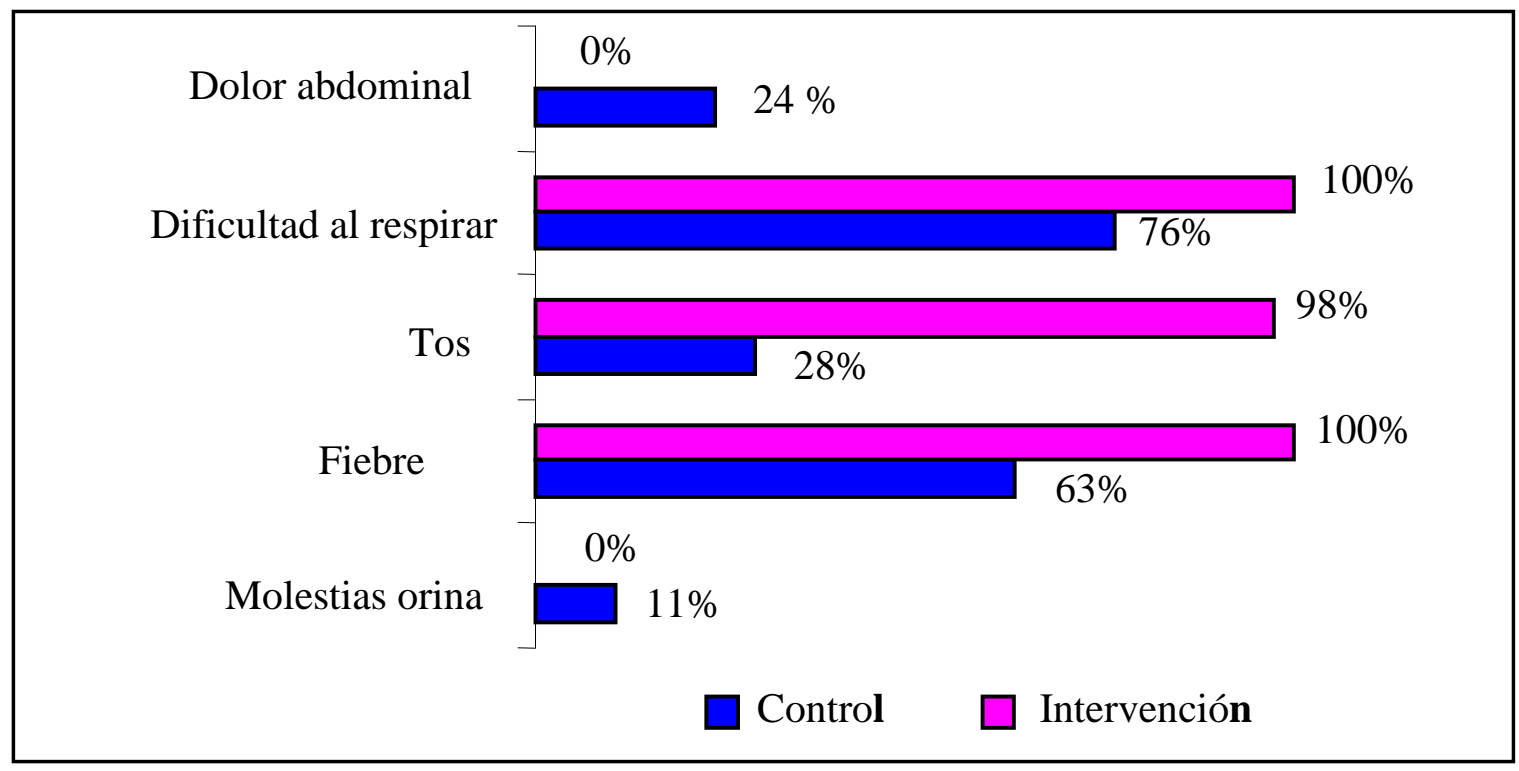

\section{DISCUSIÓN}

El presente estudio demuestra la eficacia de este programa educativo de intervención en forma de tríptico, dirigido a pacientes ingresados con neumonía en tres unidades de hospitalización, sin objetivarse diferencias estadísticamente significativas entre ellas.

Referente al nivel educacional de los pacientes, el tríptico no ha sido ningún obstáculo para que el grupo intervención entendiera la información escrita.

Cuando comparamos los pacientes que recibieron el programa educativo con aquéllos que no lo recibieron, comprobamos que los resultados obtenidos en los pacientes a los que no se les realizó ningún tipo de intervención, los conocimientos que tenían respecto a la neumonía eran mucho más bajos.

Dedicar tiempo a un paciente con neumonía para informarle de su patología, aporta muchos beneficios a todas las partes implicadas en el proceso de la enfermedad, ya que este colabora mejor en el cumplimiento terapéutico. 
Consideramos que la importancia de la información facilitada por escrito, con un lenguaje claro, conciso y asequible para las diferentes edades en pacientes ingresados, aumenta los conocimientos sobre su enfermedad, sus síntomas y las pruebas, por tanto el paciente comprende mejor su situación durante el ingreso y se muestra más relajado y cómodo ${ }^{11}$.

\section{BIBLIOGRAFÍA}

1. File TM Jr. Community-acquired pneumonia. Lancet 2003;362:1991-2001.

2. Bayas J. M, Vilella A, San-Martin M, Gonzalez A, Conesa A, Asenjo M.A. Impacto sanitario de los ingresos por neumonía en un hospital de tercer nivel. Med Clin 2001;116:694-695.

3. Dean NC. Use of prognostic scoring and outcome assessment tools in the admission decision for community-acquired pneumonia. Clin Chest Med 1999;20:521-9.

4. Almirall J, Bolíbar I, Vidal J, Sauca G, Coll P, Niklasson B, et al.. Epidemiology of community-acquired pneumonia in adults: a population-based study. Eur Respir $\mathrm{J}$ 2000;15:757-63.

5. Blanquer Olivas J. Neumonía adquirida en la comunidad del adulto que precisa ingreso hospitalario. Ars Médica.2004. Madrid.

6. Ortiz, Z. Un paciente informado, es un paciente seguro. http://www.errorenmedicina.anm.edu.ar. Enero 2007. IIE. Academia Nacional de Medicina, Buenos Aires.

7. Alfageme, I., Aspa, J., Bello, S. y cols. Normativa para el diagnóstico y tratamiento de la neumonía adquirida en la comunidad. Sociedad Española de Neumología y Cirugía Torácica (SEPAR). Arch Bronconeumol 2005; 41: 272-289.

8. Bembibre L., Lamelo F. Neumonía adquirida en la comunidad. Guías Clínicas 2004; 4 (37). fisterra.com. Disponible en www.fisterra.com/guias2/neumonia.asp.

9. Blanquer J., Alós J. I., Álvarez L. .et al. Neumonía Adquirida en la Comunidad del adulto que precisa ingreso hospitalario. Barcelona: Ars Medica, Medical / Nursing, cop. 2005. ISBN: 8497510569.

10. Prieto L, La elaboración de un cuestionario. FMC, 03 2000; Vol 7 Num 3: 138-147.

11. Instituto Europeo de Salud y Bienestar Social, La Satisfacción de los Pacientes. 2008; JANO.es y agencias. 


\section{ANEXO}

\section{ENCUESTA NEUMONÍA}

Edad......

Sexo.......

1-- Nivel de estudios de la persona que responde

Ninguno

Primarios (EGB-ESO)

Formación profesional

Bachillerato

Universitarios

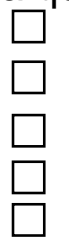

2-- ¿Es la primera vez que tiene neumonía?

$\mathrm{Si}$

No

3-- ¿Le han explicado lo que es la neumonía?

\section{$\mathrm{Si}$}

No

4-- Responda a esta pregunta si le dieron el tríptico. ¿Entendió la información que le dieron en forma de tríptico sobre la enfermedad que usted padece. (neumonía)?

Mucho

Bastante

Poco

Muy poco

Nada

5-- La información que le han dado sobre su enfermedad (neumonía) y pruebas le parece adecuada.

Mucho

Bastante

Poco

Muy poco

Nada

6-- ¿Qué cree usted que es la neumonía?

Un trombo en el pulmón

Una obstrucción en el pulmón

Una infección en el pulmón

Agua en el pulmón

Nada

7-- ¿Cual de estos síntomas aparece en la neumonía? (Hay varias respuestas ciertas)

Molestia al orinar

Fiebre

Tos

Dificultad al respirar

Dolor abdominal 